\title{
BMJ Protocol for a qualitative study of OPen knowledge translation in a participatory research project
}

\author{
Ida Lillehagen, Nina Vøllestad, Kristin Heggen, Eivind Engebretsen
}

To cite: Lillehagen I, Vøllestad N, Heggen K, et al. Protocol for a qualitative study of knowledge translation in a participatory research project. BMJ Open 2013:3:e03328.

doi:10.1136/bmjopen-2013003328

- Prepublication history for this paper is available online. To view these files please visit the journal online (http://dx.doi.org/10.1136/ bmjopen-2013-003328).

Received 31 May 2013 Revised 24 July 2013 Accepted 26 July 2013
Department of Health Science, Institute of Health and Society, University of Oslo, Oslo, Norway

\section{Correspondence to} Ida Lillehagen; ida.lillehagen@medisin.uio.no

\section{ABSTRACT \\ Introduction: In this article, we present a methodological design for qualitative investigation of knowledge translation (KT) between participants in a participatory research project. In spite of a vast expansion of conceptual models and frameworks for conducting KT between research and practice, few models emphasise how KTs come about. Better understanding of the actions and activities involved in a KT process is important for promoting diffusion of knowledge and improving patient care. The purpose of this article is to describe a methodological design for investigating how KTs come about in participatory research.}

Methods and analysis: The article presents an ethnographic study which investigates meetings between participants in a participatory research project. The participants are researchers and primary healthcare clinicians. Data are collected through observation, interviews and document studies. The material is analysed using the analytical concepts of knowledge objects, knowledge forms and knowledge positions. These concepts represent an analytical framework enabling us to observe knowledge and how it is translated between participants. The main expected outcome of our study is to develop a typology of KT practices relevant to participatory research.

Ethics and dissemination: The project has been evaluated and approved by the Norwegian Social Science Data Services. Informed consent was obtained for all participants. The findings from this study will be disseminated through peer-reviewed publications and national and international conference presentations.

\section{INTRODUCTION}

Many professional practitioners and researchers in health sciences share a concern about insufficient translation of knowledge between research and practice. Central to this concern is that lack of knowledge translation (KT) may lead to underuse of efficacious treatment, incorrect use of treatment and excessive use of inefficacious or unevaluated treatment and thus deprive patients of optimal healthcare. This has led to a wide effort to facilitate

\section{ARTICLE SUMMARY}

Article focus

- A primary objective is to improve our understanding of how knowledge translations come about.

- We aim at examining the microlevel practices of knowledge translation between different participants in clinical research.

- Meetings discussing all aspects of a participatory research programme are studied using qualitative methods.

Key messages

- Knowledge translations can be observed as shifts in and among knowledge objects, knowledge forms and knowledge positions.

- Barriers and facilitators for knowledge translation can be identified.

- A typology of situations where knowledge translation is performed in different ways is an expected outcome.

Strengths and limitations of this study

- A multidisciplinary team of researchers, including competence in qualitative methods and in clinical science, provides access to all relevant areas to allow adequate analyses.

- The design provides possibilities for validation of analyses.

- One particular research group is studied, and therefore the findings cannot directly be extrapolated to other settings or generalised.

practice-relevant research and researchinformed practice decisions. ${ }^{1}{ }^{2}$ Numerous frameworks and conceptual models have been developed. ${ }^{3}{ }^{4}$ Participatory research is one strategy for optimising translation of knowledge between representatives from research and clinical practice, based on the assumption that promoting collaboration between researchers and practitioners improves the relevance of the results. ${ }^{5}$ Despite positive evaluations of face-to-face interaction between researchers and practitioners, ${ }^{6} 7$ little is known about how KT is actually performed in these settings. ${ }^{8}$ This paper presents an outline of an 
ethnographic investigation of KTs in a participatory research programme focusing on the practice of KT, that is, the concrete actions and activities involved in translating knowledge between researchers and clinicians in face-to-face interaction.

A much used model which conceptualises the relationship between research and practice is the so-called translational chain, consisting of three steps: from basic science to human application (T1), from human to proven clinical application, for instance through randomised controlled clinical trials (T2) and from clinical studies to routine clinical practice (T3) ${ }^{9}$ Another model is the Knowledge-to-Action-Model developed by the Canadian Institute of Health Research that postulates a knowledge creation pyramid with three stages (knowledge inquiry, knowledge synthesis and knowledge tools) as well as an application cycle. ${ }^{10}$ Common to most existing KT models is that they primarily illustrate what a translational process or intervention consists of and not how translation comes about. ${ }^{11}$ KT research thus mainly focuses on implementing certain knowledge products and evaluating outcome in terms of changing behaviour of (mostly) healthcare providers. ${ }^{12-14}$ Some studies focus on KTs as events that involve interaction between human and non-human actors. ${ }^{11} 1516$ These studies illustrate how KTs are not solely technical processes of implementing preconstructed packages of information, but processes that are socially accomplished through interaction. Although tending to focus on the actors involved, that is, practitioners, knowledge brokers and implementation researchers, these articles highlight the need to study KTs at a microlevel.

Our approach is innovative in at least three ways: (1) it emphasises KT as concrete microlevel practices which are often marginalised or black-boxed in existing KT models and research. (2) Instead of focusing on the roles of the individuals present, it focuses on the many situations in which knowledge is presented, discussed and negotiated between the participants in the meetings (3) It proposes a way to operationalise the rather abstract notion of 'knowledge translation' by breaking it down to shifts in and between three observable phenomena, that is, knowledge positions, knowledge objects and knowledge forms.

\section{Our arena of study}

Our chosen case is the research programme FYSIOPRIM which has been established to meet some of the challenges for physiotherapy research in primary healthcare. ${ }^{17}$ The programme includes several clinical research projects and also studies of methods to be applied in clinical practice and research. FYSIOPRIM is built on a close collaboration between researchers and primary healthcare clinicians. In order to secure research and methodological development that are relevant for clinical practice, regular and formal meetings are organised between clinicians and researchers. These meetings (coordinators' meetings) are arenas for translation of knowledge between those representing research and those representing clinical practice. The researchers are those who summarise discussions and develop background material that is sent to the clinicians before the meetings. They also introduce the topic to be discussed and thereafter open up for input and views from the clinicians. Later on, the researchers summarise the discussions and revise the plans accordingly. The same topic may be discussed repeatedly before the plans are finalised. FYSIOPRIM can thus be seen as an example of a participatory research project emphasising a bottom-up approach, locally defined priorities and local perspectives. ${ }^{18}$ It is a research project in which 'local knowledge and perspectives are not only acknowledged but form the basis for research and planning. ${ }^{18}$

As the aim of the coordinator meetings is to integrate knowledge from clinical practice and research, it is an a priori assumption that these differ from each other. The clinicians are, for instance, expected to present knowledge about what kind of treatments they use for different patients and their rationale for doing so. The researchers are expected to present the background for their research hypothesis or design, for example the rationale for a clinical trial.

The project described in this protocol is a metastudy of the coordinator meetings and the KT involved.

\section{METHODS AND ANALYSIS Objective}

The objective of the study is to investigate how KTs take place in the interaction between participants in a participatory research project. The overall research question is how knowledge is translated between researchers and practitioners in the coordination meetings for FYSIOPRIM.

\section{Collection of data}

Our study combines several methods including (1) observation of meetings, (2) reflection meetings, (3) interviews and (4) document studies.

\section{Observations}

About 15 meetings will be observed from the beginning to the end of the project period in order to capture the different phases of the project: planning, analysis, interpretation and writing up results. Three researchers will be involved in the data collection. One observational researcher (the first author) will be present at every meeting. In addition, two experienced field researchers (author numbers three and four) will alternately be present at every third FYSIOPRIM meeting. Organising the data collection with the use of three observers is anticipated to give a rich material as well as contributing to the validation of data. Our assumption is that being present as a researcher adds value beyond listening to a tape recorder. Getting a feel of the ambience among the participants-and what Goffman ${ }^{19}$ refers to as rules of 
conduct in the particular setting-might add valuable information.

Meetings will be recorded for their entire duration, transcribed verbatim and complemented by the observational researchers' notes. Accuracy in transcriptions and close attention to language will be necessary, since KT involves practices that are largely linguistic in nature. KT happens through speech acts such as summarising, repeating, questioning, rephrasing or arguing and the use of various changing words to denote and emphasise meaning. In order to capture these and the participants' talk as situated social action, we will use Jefferson's system of transcription, which shows the precise beginning and ending of overlapping talk, intonation aspects and timed pauses. ${ }^{20}$ We will also use a field diary to record activities and interactions. The aim of the field diary is primarily to capture what the recordings do not provide. Notes may consist of observations of non-verbal aspects such as body language and how material objects such as technology or documents are used. ${ }^{21}$

Although the ethnographic methods used are rather traditional, our interest in KT practices calls for a different focus than that of many other ethnographic studies (this approach will also guarantee the participants' anonymity). Inspired by microethnographic studies, we are concerned with the situations produced in the interaction between individuals rather than the individuals themselves. ${ }^{22}$ Our interest is not in how participants manage their professional expertise, but in the sensemaking process. The interaction process is at the centre of our study. Examples of questions and topics guiding the data collection are as follows: What sort of topics and dilemmas do the participants take a common interest in? Who are responding to what sort of questions in various situations, in what way and with what effect? What sort of arguments are raised as the participants reflect, discuss and try to define a particular problem? What problem-solving approaches are suggested with what effect? When and how is an argument legitimised in clinical experience, scientific evidence or both? How is coherence between research evidence and clinical practice argued? When are missing links between research and practice a problem of concern? What sort of examples and arguments provide opportunities for meaningful engagement from whom? When do two or more of the participants commonly trigger disagreement with an initial statement?

\section{Reflection meetings}

Immediately after a coordinators' meeting, the observational researcher sends a résumé of her observations/preliminary analysis to the PI of FYSIOPRIM and the co-researchers. A meeting is then set up (reflection meeting) as soon as possible and no later than 1 week after the observations in order to discuss the résumé. The purpose of the reflection meetings is twofold: (1) an analytical purpose: The researchers and the PI of FYSIOPRIM discuss possible interpretations of the observed situations.
The observational researcher's résumé forms the basis for the discussion, as well as the co-researcher's and the PI's reflections and notes. (2) A dissemination purpose: the three researchers present their preliminary findings to the PI of FYSIOPRIM.

Based on the preliminary findings, the PI may plan the possible steps in order to improve KT in the future meetings and other activities. However, the three researchers do not take part in this action planning. The PI of FYSIOPRIM writes a short résumé of actions taken which is handed over to the researchers before the next coordination meeting. Changes made by the PI based on preliminary findings might have an influence on KT among the participants in FYSIOPRIM. However, such influence is not seen as problematic, but as a facilitator of new forms of KT between the participants, which in turn will be subject to further observation.

\section{Interviews}

We will also conduct supplementary interviews with the participants. At least 10 interviews will be conducted (5 researchers, 5 clinicians). Re-interviewing or more interviews might become necessary. The observational researcher will be conducting the interviews together with the two co-researchers who will be present in two interviews each. Interviews will be organised in relation to (before or after) FYSIOPRIM meetings. All interviews will be tape recorded and transcribed. Transcription will include conversation, yet exclude utterances like uh, ah and intonation. Pauses will be marked, but not timed.

Interviews will not follow a common interview guide but questions will be developed continuously for each interview, based on observations and reflection meetings. However, the interviews will be organised based on the following general topics: (1) we will ask the participants to describe situations which they have found particularly interesting and/or challenging. We will further discuss the characteristics of these situations and elaborate on the barriers to and facilitators of knowledge translation. (2) The researchers will present situations for the participants that have struck them as particularly interesting and/or challenging with regard to the translation of knowledge. Such a situation might be an utterance that seems to change the direction of the overall discussion or, in contrast, an utterance that seems to be marginalised or ignored. The interviewees will also be asked to comment on the researchers' preliminary analysis. Characteristics of the selected situations as well as barriers and facilitators for KT will be discussed.

The aim of the interviews is threefold: (1) preliminary analysis of data through discussion of selected sequences/situations (2) validation in terms of adding information and (3) providing additional data for further analysis. $^{23}$

\section{Documents}

Much of the interaction in the group is based on written documents. These comprise journal papers (or extracts 
from them), documents such as drafts of research plans, suggestions for questionnaires to be used, data collection plans, etc. In addition, results of the ongoing research or papers recently written may also be distributed. The documents are most often produced by the researchers and may be sent out as a foundation for the discussions in the coordinators' meeting. The documents may be modified as a result of the discussions and redistributed in updated versions. Hence, the documents cannot be seen as passive reflections of events, but as actors taking part in the situational interplay. ${ }^{24}$ Furthermore, the documents capture and preserve processes of change throughout the data collection period and reflect in this way a translation history within the group.

\section{Analysis of data}

Analytical tool

One of the reasons why the practice of KT is poorly understood may be that KT is a rather abstract notion. Not unlike concepts like 'discussion' or 'reflection', KT refers to a form of activity that is difficult to observe. We know that we are doing it, but we do not know what we do when we are doing it. In the first 6 months of our project (the pilot phase), we have developed analytical tools that may enable us to open this black-box. The tool development is partly based on observations and preliminary analysis and partly on a reading of the theoretical literature. The purpose of our analytical tools is to operationalise KT research by breaking it down into observable entities.

In our further analysis, we have decided to concentrate on three aspects of knowledge: (1) knowledge objects, (2) knowledge forms and (3) knowledge positions. While a knowledge object refers to the immediate topic or piece of content addressed by someone intending to create or communicate knowledge (a muscle function, an experience of pain, etc), the knowledge form refers to ways of organising or communicating this knowledge (theories, prognosis, probabilistics, examples, anecdotes, questions, etc). The knowledge position is the point of view from which the knowledge is created or used (clinician position, basic/clinical researcher position, policy-maker position, patient position, etc)

The concept of knowledge objects is influenced by Annemarie Mol's ${ }^{25}$ study of ontology in medical practice. Mol demonstrates that the objects of knowledge vary with the practices in which they are manipulated. Her 'atherosclerosis' case is, for instance, a slightly different phenomenon depending on specialty or treatment. According to Mol, a disease, a condition or a body part is not one 'object in the middle waiting to be observed'; rather, it represents a multiplicity of realities or substances depending on the many practices related to it. Our perspective is also inspired by studies which have pointed to the diversity of 'worlds' existing within scientific and clinical practice. ${ }^{162325}$ This illustrates how the object or 'world' in question will necessarily be different or alter through the translational chain, depending on the practice with which it is handled. Translation thus implies repeated shifts between objects. In the observed discussions, the researchers may, for instance, present a test procedure based on research showing that motor control patterns are altered in patients with neck pain. The clinicians might claim that the procedure does not test the phenomena they believe are of importance. Rather, they would focus on the patient's fear avoidance behaviour as a limiting factor. In our study, we focus on how the participants manage to integrate objects as different as individual pain, joint function and basic functions in their talk and still maintain coherence in discussions.

The concept of knowledge forms is influenced by rhetoric of science and more precisely the concept of topos drawn from classical rhetoric. ${ }^{26}$ Topos originally means 'place' and is to Aristotle a general argument base or template on which individual arguments are built. Some examples highlighted by Aristotle are definitions, induction, consequences and division. ${ }^{26}$ When we present our knowledge in scientific articles or in face-to-face communication with peers, we also build on templates or structures that make our arguments credible or recognisable as knowledge to the reader or listener. ${ }^{27}$ Which forms are accepted or recognised may vary radically from one knowledge culture to another. For qualitative researchers, a case analysis may, for instance, be an adequate knowledge form, while many quantitative researchers primarily express their knowledge through probabilities. When it comes to clinicians, anecdotes of individual patients may qualify as knowledgeable.

Our third analytical concept, knowledge position, is inspired by Karin Knorr-Cetina's ${ }^{23}$ theories on epistemic cultures. In Knorr Cetina's studies, these are cultures related to scientific practice; different scientific practices exhibit different epistemic cultures. Scientific practice in our case is a means not only to create knowledge, but to include all practices involved in the KT chain. In encounters between different epistemic cultures, some positions are possible while others are excluded. Positions are different from roles because they are not tied to the individual. They belong to the situation rather than the person. The situations decide which positions are possible for the participant to take, not individual characteristics or choices. This means that knowledge positions such as teacher/student, expert/ non-expert or even researcher/practitioner are flexible and altering entities that may shift from one situation to the other.

To perform KT implies altering some or all of these three aspects of knowledge. By analysing shifts in and between them, we might be able to articulate the practices involved in KT.

\section{Analytical approaches}

The researchers will organise the knowledge objects, forms and positions into categories using a variety of 
methods. For instance, data modelling in NVivo V.10 $10^{28}$ will be used to code the data into the three domains, in search of variations within and between them. ${ }^{28}$ The observational researcher (first author) will have responsibility for this software supported first phase of the analysis. This analysis will then be combined with rhetorical analysis, which is a close reading approach, where author number four will have the main responsibility and work partly together with the observational researcher. The aim of this analysis is to capture the forms (topoi) used in presenting the knowledge objects, as well as which rhetorical techniques are used to relate objects and forms to a specific position. ${ }^{29}$ As part of our close reading, we will first annotate the texts, that is, highlight key words, phrases or anything else that strikes us as significant. Second, we will go deeper into the specifics, drawing on text-analytical and especially narratological tools. ${ }^{30} 31$ Some of the aspects that will be analysed are: the point of view (narrative perspective and voice) required to identify the knowledge positions, ${ }^{31}$ the choice of words and imagery (eg, metaphors), ${ }^{32}$ as well as structure, for example, the interplay between different genres $^{27}$ in order to capture the knowledge forms and description/characterisation in order to identify the objects in question. ${ }^{31}$

It will be organised into three types of meetings in order to develop the analysis and test the reliability of the findings. (1) Regular and frequent meetings between the three researchers. (2) Meetings between the three researchers and PI in FYSIOPRIM. (3) One extensive meeting with all participants in FYSIOPRIM where the findings will be presented and discussed. In addition, drafts of papers presenting results from the KT project will be sent to all participants in FYSIOPRIM in order to get comments as well as to exert extracontrol of their informed consent.

\section{Possible outcome of the analysis}

If our study is successful, it will provide a typology of KT practices. A hypothetical example may illustrate what we expect to gain from our study.

Imagine that the research team presents a draft list of items to be used to generate a database of patient profiles and treatment in primary care physiotherapy. The responsible researcher invites the clinicians to comment on the clinical applicability of the items and to suggest alternative items or wording. One of the clinicians answers by commenting on a question about sex life. The clinician claims that the question, although providing important information for statistical purposes, might be conceived as offensive and thus be detrimental to communication with the patient. She/he tells a story about a situation in which she/he has experienced this.

Out of many possible responses from the researcher, one might imagine three: (1) she/he might stress that the information provided by the question is crucial for statistical purposes and ask the clinician if she/he could formulate the question in another way that is less offensive; (2) she/he might respond by saying that the clinician's comment illustrates that the database has a dual function: to provide information for statistical purposes (research, patient profiles for clinical use) and to facilitate communication. This specific question might thus represent a conflict of interest, and the researcher might invite the group to discuss how to take both these considerations into account and (3) she/he might suggest deleting the question.

In the first hypothetical response, the researcher insists on the knowledge object being an information tool to be used for statistical purposes. She/he also insists on the knowledge form she/he expects from the clinician: a (reformulated) question providing this information. She/he is not willing to move from a position which prioritises statistical purposes, such as optimising the possibilities for comparing results to other studies.

In the second hypothetical response, the researcher is willing to negotiate about the knowledge object. Unlike the first response, the question or the object alters from being regarded as an information tool into being an information and a communication tool. She/he neither insists on a particular knowledge form nor expects the clinician to come up with an alternative question, but invites different kinds of input from the group in terms of a broad invitation for 'discussion'. Rather than insisting on the position of the statistical user, she/he tries to establish a common position where the participants as co-researchers must jointly decide how to tackle the conflict of interest between getting information and facilitating communication.

In the third hypothetical response, the researcher lets the knowledge object, form and position presented by the clinician settle the matter.

The point here is not that one of the responses is the right one. That depends on contextual factors, like the aim of the cooperation. The point is, however, that the three responses are different and might be said to constitute different KT practices. In the first situation, translation consists of incorporating the input of the clinician into the researcher's knowledge culture or system. KT is integration or incorporation. In the second case, translation is a process that implies creating something new together. The outcome of the translational process is at least to a certain degree common knowledge objects, forms or positions. Translating is sharing or cocreating. In the third and last case, the voice of the clinician is dominant. Translation is the same as adopting the other's position.

Through our study, we might be able to provide a classificatory system for categorising differences and similarities, as well as shifts in and between knowledge objects, forms and positions that can be used as a typology of situations where KT is performed in different ways.

\section{ETHICS AND DISSEMINATION}

The informants have been given information about the project orally as well as in a written informed consent letter. 
The project is based on studies of a research programme known to the public and the identity of the participants may be easily found. However, publications and presentations from the study will present findings anonymously. Excerpts or statements will be anonymised using terms such as participant 1, 2, etc. Identity issues were discussed with the participants during revisions of the written informed consent letter. It was emphasised that results should be presented in a manner that focuses on the translation of knowledge between participants and avoids any focus on individuals.

The project is exempted from ethical evaluation by Regional Committees for Medical and Health research ethics as it does not involve health-related research on human participants. The project has received ethical approval from the Norwegian Social Science Data Services. Results from this study will be published in peer-reviewed scientific journals and presented at one or more scientific conferences.

Acknowledgements The authors would like to thank Molly Sutphen and Tom Sanders who have contributed to the development of the methodological design as well as with valuable manuscript revision.

Contributors IL, NV and EE drafted the manuscript. KH helped to draft and extensively revise the manuscript. All authors have approved the final manuscript and are willing to take responsibility for appropriate portions of the content.

Funding This work was supported by the University of Oslo.

Competing interests None.

Provenance and peer review Not commissioned; internally peer reviewed.

Open Access This is an Open Access article distributed in accordance with the Creative Commons Attribution Non Commercial (CC BY-NC 3.0) license, which permits others to distribute, remix, adapt, build upon this work noncommercially, and license their derivative works on different terms, provided the original work is properly cited and the use is non-commercial. See: http:// creativecommons.org/licenses/by-nc/3.0/

\section{REFERENCES}

1. Woolf $\mathrm{SH}$. The meaning of translational research and why it matters. JAMA 2008;299:211-13.

2. Zerhouni EA. Translational and clinical science. Time for a new vision. N Engl J Med 2005;353:1621-3.

3. Bero L, Grilli R, Grimshaw J, et al. Closing the gap between research and practice: an overview of systematic reviews of interventions to promote the implementation of research findings. The Cochrane Effective Practice and Organization of Care Review Group. BMJ 1998;317:465-8.

4. Scott S, Albrecht L, O'Leary K, et al. Systematic review of knowledge translation strategies in the allied health professions. Implement Sci 2012;7:70.

5. Mitton C, Adair CE, McKenzie E, et al. Knowledge transfer and exchange: review and synthesis of the literature. Milbank $Q$ 2007;85:729-68.
6. Kothari A, Birch S, Charles C. "Interaction" and research utilisation in health policies and programs: does it work? Health Policy 2005;71:117-25.

7. Ross S, Lavis J, Rodriguez C, et al. Partnership experiences: involving decision-maker in the research process. J Health Serv Res Policy 2003;8:26-34.

8. Ginsburg L, Lewis S, Zackheim L, et al. Revisiting interaction in knowledge translation. Implement Sci 2007;2:34.

9. Dougherty D, Conway PH. The "3T's" road map to transform US healthcare. JAMA 2008;299:2319-21.

10. Graham I, Logan J, Harrison M, et al. Lost in knowledge translation: time for a map? J Contin Educ Health Prof 2006;26: 13-24.

11. Kitto SC, Sargeant J, Reeves S, et al. Towards a sociology of knowledge translation: the importance of being dis-interested in knowledge translation. Adv Health Sci Educ Theory Pract 2012;17:289-99.

12. Baker R, Camosso-Stefanovic J, Gilliss C, et al. Tailored interventions to overcome identified barriers to change: effects on professional practice and health care outcomes. Cochrane Database Syst Rev 2010;2:CD005470.

13. Eccles M, Grimshaw J, Walker A, et al. Changing the behavior of healthcare professionals: the use of theory in promoting the uptake of research findings. J Clin Epidemiol 2005;58:107-12.

14. Davis $\mathrm{D}$, Thomson $\mathrm{M}$, Oxman $\mathrm{A}$, et al. Changing physician performance: a systematic review of the effect of continuing medical education strategies. JAMA 1995;274:700-5.

15. Rowley E. Protocol for a qualitative study exploring the roles of 'Diffusion Fellows' in bridging the research to practice gap in the Nottinghamshire, Derbyshire and Lincolnshire Collaboration for Leadership in Applied Health Research and Care (CLAHRC-NDL). BMJ Open 2012;2:e000604.

16. Moreira T. Diversity in clinical guidelines: the role of repertoires of evaluation. Soc Sci Med 2005;60:1975-85.

17. Vøllestad N. Primary care research-new opportunities. Public service review. Eur Sci Technol 2011:110-11.

18. Cornwall A, Jewkes $\mathrm{R}$. What is participatory research? Soc Sci Mede 1995;41:1667-76.

19. Goffman E. Interaction ritual: essays in face-to-face behavior. New Brunswick, NJ: Aldine Transaction, 2005.

20. Jefferson G. Glossary of transcript symbols with an introduction. In: Lerner $\mathrm{GH}$, ed. Conversation analysis studies from the first generation University of California. Santa Barbara: John Benjamins Publishing Company, 2004.

21. Sidnell J, Stivers T. The handbook of conversation analysis. Chichester, West Sussex: Wiley-Blackwell, 2013.

22. Fitch KL, Sanders RE. Handbook of language and social interaction. Mahwah, NJ: Lawrence Erlbaum Associates, 2005.

23. Knorr-Cetina K. Epistemic cultures: how the sciences make knowledge. Cambridge, MA: Harvard University Press, 1999.

24. Latour B, Woolgar S. Laboratory life: the construction of scientific facts. Princeton, NJ: Princeton University Press, 1986.

25. Mol A. The body multiple: ontology in medical practice. Durham: Duke University Press, 2002.

26. Mendell H. Topoi on Topos: The development of Aristotle's concept of place. Phronesis. JSTOR 1987;32:206-31.

27. Bauman R. A world of others' words: cross-cultural perspectives on intertextuality. Malden, MA: Blackwell Publishing, 2004.

28. QSR International Ltd. QP. NVivo version 10. 2012.

29. Toulmin S. The uses of argument. Cambridge: Cambridge University Press, 2003.

30. Bal M, Boheemen CV. Narratology: introduction to the theory of narrative. Toronto: University of Toronto Press, 2009.

31. Genette G. The architext: an introduction. Berkeley, California: University of California Press, 1992.

32. Lakoff G, Johnson M. Metaphors we live by. Chicago: University of Chicago Press, 2003 